\title{
Reverse Direction Transmissions and Network Coding for Energy-Efficient Wi-Fi Networks
}

\author{
Raul Palacios ${ }^{\mathrm{a}}$, Dzmitry Kliazovich ${ }^{\mathrm{b}}$ and Fabrizio Granelli ${ }^{\mathrm{a}}$ \\ a \{palaciostrujillo,granelli\}@disi.unitn.it, University of Trento, Trento, Italy \\ bdzmitry.kliazovich@uni.lu, University of Luxembourg, Luxembourg
}

\begin{abstract}
This paper proposes a combination of the Reverse Direction Protocol (RDP) defined in the IEEE 802.11n and the Network Coding (NC) technique to improve the energy efficiency of wireless networks based on the IEEE 802.11. The aim of the combined approach, named BidCode, is to allow intermediate nodes to combine several received packets into coded packets and immediately forward them upon successful reception of data. The energy efficiency of BidCode is analyzed in this paper and compared to those of the Distributed Coordination Function (DCF) and an important NC protocol based on IEEE 802.11, called COPE. Both analytical and simulation results show the high energy efficiency of BidCode with gains of up to $350 \%$ and $130 \%$ when compared to DCF and COPE, respectively.*
\end{abstract}

\section{INTRODUCTION}

The Network Coding (NC) paradigm has been widely recognized as a powerful mean for improving energy efficiency in wireless networks [1]. The idea behind the NC approach is to allow intermediate nodes to simultaneously transmit combined information from several sources to multiple destinations by exploiting the broadcast channel. This operation implies a reduction of the total number of channel accesses, which results in less energy consumed per delivered bit of information.

Despite the potential energy savings of $\mathrm{NC}$, the inspiring work in [2] showed that there exist important practical considerations that should be taken into account for the proper implementation of $\mathrm{NC}$ in currently operating wireless networks. In particular, NC awareness of the Medium Access Control (MAC) protocol is essential to allow assigning different access priorities to multiple nodes based on the NC operation. Unfortunately, the widely used Distributed Coordination Function (DCF) of the IEEE 802.11 standard [3] does not represent a suitable MAC solution as it was designed to provide equal channel access opportunities for all the nodes on average.

To illustrate this problem, Fig. 1 shows two simple, but important and widely employed, network topologies, namely, (a) Alice and Bob and (b) cross. In Alice and Bob topology (see Fig. 1a), relay node $R$ would always capture $1 / 2$ of the channel to send twice more data packets than source nodes $A$ and $B$. However, $R$ will get $1 / 3$, due to the DCF fairness, when $A$ and $B$ increase their transmission rates, which together will capture up to $2 / 3$ of the channel. Therefore, $R$ will not be able to forward packets to $A$ and $B$ with the same rate as they arrive. On the contrary, when $R$ enables NC operations, it uses $1 / 3$ of the channel to send coded packets

\footnotetext{
*This work is funded by the GREENET project (PITN-GA-2010-264759).
}

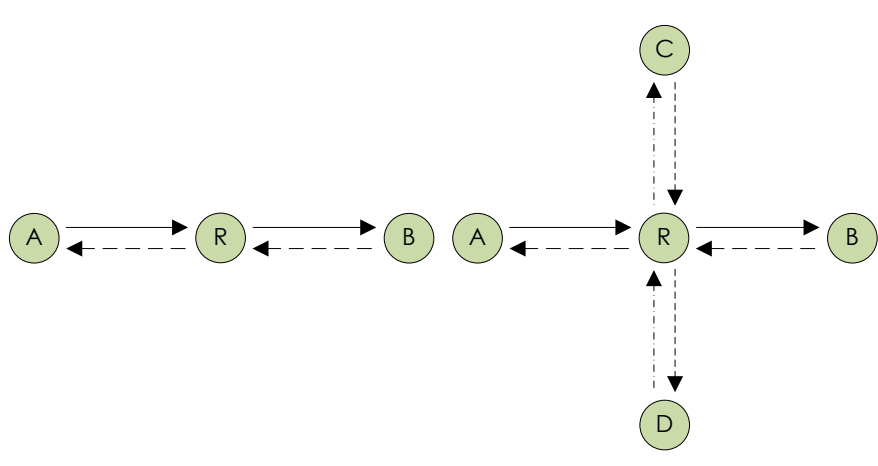

(a) Alice and Bob.

(b) Cross

Fig. 1. Reference scenarios: (a) Alice and Bob, wherein two source nodes $A$ and $B$ exchange data through relay node $R$ and (b) Cross, wherein two bidirectional flows from $A$ and $B$ and $C$ and $D$, respectively, intersect at $R$.

that contain pairs of packets from $A$ and $B$, reaching $2 / 3$ and thus matching the incoming and outgoing rates. In cross topology (see Fig. 1b), $R$ gets $1 / 5$ of the channel, because there are four source nodes around it, while it is receiving packets from $A, B, C$, and $D$ with $4 / 5$ rate. Even though NC is enabled, $R$ can only send a coded packet composed of a pair of packets from $A$ and $B$ or $C$ and $D$, respectively, reaching $2 / 5$ which is not sufficient to match the incoming rate.

In order to cope with this limitation, the relay nodes that are ready to transmit coded packets could be granted higher priority for channel access, as coded packets can provide more information for the network than non-coded packets. For this, previous works [4]-[7] proposed to adjust the size of the Contention Window (CW) based on the level of traffic congestion, the state of channel contention, and NC information. However, in these works, the relay nodes are still affected by the serious competition of all nodes for channel access, since the access priority is higher on average, i.e., probabilistic. Consequently, the proposed schemes cannot guarantee an immediate channel access for the relay nodes when they actually need it.

In our previous work [8], we presented a new NC-aware MAC protocol, coined BidCode, that allows bidirectional transmissions involving network coded data packets between wireless nodes. BidCode is an extension of our previously proposed Bidirectional MAC (BidMAC) [9], [10] inspired by the already standardized Reverse Direction Protocol (RDP). In both BidMAC and RDP, the receiving node of a data packet can respond with a data packet, instead of just sending an explicit acknowledgment (ACK). However, in RDP the initiator 
of the reverse direction transmission is the transmitting node whereas in BidMAC it is the receiving node, which can extend the transmission duration based on the amount of data to send.

The performance evaluation of BidCode [8] was based on computer-based simulations considering the Alice and Bob scenario. In [11], we analyzed the upper bounds of BidCode throughput and energy efficiency in both the Alice and Bob and cross scenarios and produced performance evaluation using numerical results only.

In this paper, we present a comprehensive performance evaluation of BidCode in terms of energy efficiency via analysis and computed-based simulations in both the Alice and Bob and cross topologies. The energy efficiency results of BidCode are compared to those of the DCF, BidMAC, and a well-known $\mathrm{DCF}+\mathrm{NC}$ protocol referred to as COPE [2], considering the traffic load, packet length, and data rate.

The remainder of the paper is structured as follows. Section II provides a brief description of the evaluated protocols. In Section III, the energy efficiency analysis of the protocols is presented. The evaluation results are then discussed in Section IV. Finally, Section V concludes this paper.

\section{NC-AwARE MAC PRotocols OVERVIEW}

This section overviews the protocols under consideration, i.e., DCF, COPE, BidMAC, and BidCode, and shows an example of operation of each protocol in Fig. 2.

\section{A. $C O P E$}

COPE inserts an NC layer on top of the MAC layer that is responsible for performing linear combinations of several received packets from different flows using XOR operations. In order to allow proper NC operation, COPE introduces a number of modifications in the network stack architecture. First, nodes enable the promiscuous mode to process and store overheard packets for a limited time. Second, nodes opportunistically produce coded packets and send them to one of the intended receivers with an additional header that includes a complete list of the next-hop receivers. Third, upon successful decoding, receiving nodes schedule ACK events that are to be sent together with data packets or periodic control packets. Finally, nodes periodically inform their neighbors about the packets they have stored for coding and decoding opportunities, through reception reports.

The MAC protocol operation of COPE is according to the DCF of the IEEE 802.11. This MAC protocol employs the Carrier Sense Multiple Access with Collision Avoidance (CSMA/CA) mechanism in combination with a binary exponential backoff algorithm and an optional Request-ToSend/Clear-To-Send (RTS/CTS) handshake. As shown in Fig. $2 a$, in each data transmission, the transmitting node waits for a DCF Inter Frame Space (DIFS) and a random backoff period. Then, the transmitting and receiving nodes exchange the RTS and CTS packets, interleaved by a Short Inter Frame Space (SIFS), before the transmission of data. Upon successful reception of data, the receiving node responds with an ACK packet after a SIFS. Other nodes overhearing the exchange of

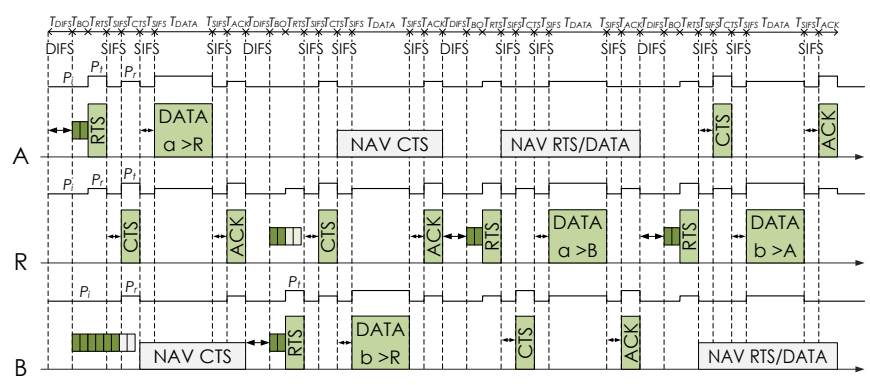

(a) DCF: $R$ forwards the packets from $A$ and $B$ to their respective destinations.

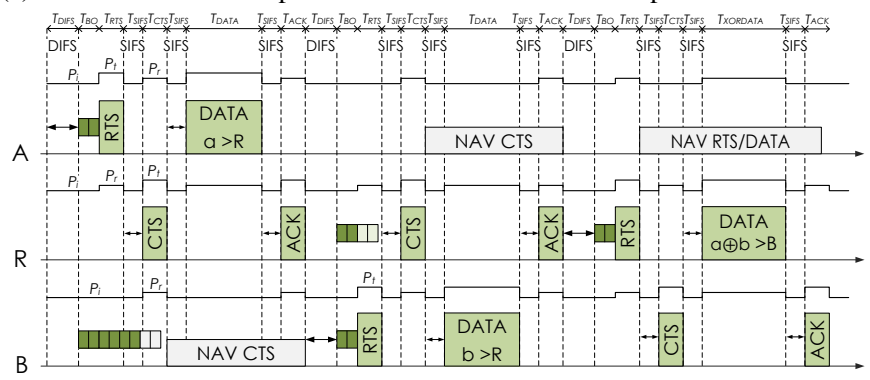

(b) COPE: $R$ encodes the packets from $A$ and $B$ and forwards a coded packet.

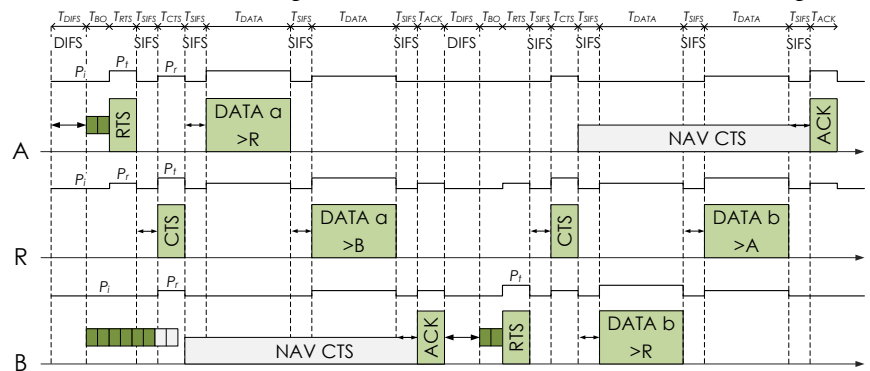

(c) BidMAC: $R$ can send a data packet immediately after receiving it.

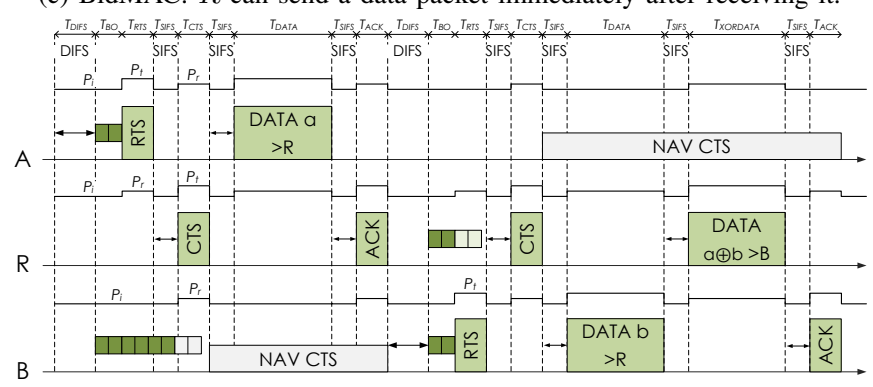

(d) BidCode: $R$ can send a coded packet upon successful data reception.

Fig. 2. Examples of operation of the DCF, COPE, BidMAC, and BidCode protocols in the Alice and Bob scenario.

packets read the duration field of control and data packets and update their Network Allocation Vectors (NAVs).

The COPE protocol operates as shown in Fig. 2b. When node $A$ seizes the channel, it sends packet $a$ to node $R$ using the RTS/CTS mechanism while node $B$ stops the backoff procedure upon overhearing the CTS packet destined to node $A$. After a DIFS, node $B$ resumes the backoff procedure and obtains a transmission opportunity earlier than node $R$. After receiving packet $b$, node $R$ combines $a$ and $b$ into $a \oplus b$, completes the backoff procedure, and randomly sends $a \oplus b$ to node $B$, which immediately replies with an ACK packet. Both nodes $A$ and $B$ can retrieve packets $b$ and $a$, respectively, by using their own packets and the received coded packet. 


\section{B. BidCode}

BidCode exploits NC as specified in COPE together with the reverse direction transmission mode defined in BidMAC. Fig. 2c illustrates the operation of BidMAC. When node $R$ receives the RTS packet from node $A$, it replies with a CTS packet whose duration field is updated with the additional time required to enable a transmission in the reverse direction. Thus, node $B$ can update its NAV with the longer duration of the transmission and node $R$ can immediately forward packet $a$ to node $B$ upon receiving it, after a SIFS. Then, node $B$ acknowledges data reception with an explicit ACK packet and node $A$ can interpret the newly received data packet as an implicit ACK packet for its transmitted data packet. In a similar way, packet $b$ is forwarded from node $B$ to node $A$.

Following the description of BidMAC, BidCode operates as depicted in Fig. 2d. Node $A$ transmits packet $a$ to node $R$ by using standard DCF rules. However, when node $R$ receives the RTS packet from node $B$, it identifies a coding opportunity with packet $a$ and sends back a CTS packet with the value of the duration field extended to cover the transmission of the possible coded packet. Then, node $B$ sends packet $b$ and node $R$ responds with $a \oplus b$. Node $B$ completes the data exchange by sending an ACK packet and both nodes $A$ and $B$ can retrieve the original packets as explained above for COPE.

\section{ENERGY EFFICIENCY ANALYSIS}

In this section, we analyze the maximum achievable energy efficiency of the protocols in a generalized scenario that includes both the Alice and Bob and cross topologies.

\section{A. System Model and Assumptions}

We consider a wireless network composed of a relay node and $\mathrm{N}$ source nodes, all equipped with IEEE 802.11n wireless interfaces. Since the analysis is aimed at computing the upper bound of the energy efficiency, we assume that the probability of collision is zero and the channel is ideal. Also, there are always packets to transmit in the buffer but no packets are lost due to buffer overflow. All data packets have a constant bit length. Fragmentation is not used and the propagation delay is neglected. In addition, we assume that the time and energy spent for XOR coding/decoding are negligible.

We choose one possible configuration of IEEE 802.11n that is equivalent to IEEE 802.11g. Specifically, the Extended Rate Physical-layer (ERP) OFDM specification is used, which provides 8 transmission modes with data rates from $6 \mathrm{Mbps}$ to $54 \mathrm{Mbps}$ and Number of Data Bits Per OFDM Symbol $\left(N_{D B P S}\right)$ from 24 to 216 . The transmission times of RTS $\left(T_{R T S}\right)$, CTS $\left(T_{C T S}\right)$, ACK $\left(T_{A C K}\right)$, DATA $\left(T_{D A T A}\right)$, and XORDATA $\left(T_{X O R D A T A}\right)$ packets are calculated by [3] as

$$
T_{x}=T_{\text {pre }}+T_{\text {sig }}+T_{\text {sym }}\left\lceil\frac{L_{\text {serv }}+8 \cdot L_{x}+L_{\text {tail }}}{N_{D B P S}}\right\rceil+T_{\text {sigEx }}
$$

where $x$ is the type of packet, $T_{\text {pre }}$ is the preamble time, $T_{\text {sig }}$ is the signal time, $T_{\text {sym }}$ is the duration of an OFDM symbol, and $T_{\text {sigEx }}$ is the signal extension period. Inside the ceiling function $\lceil\rceil,. L_{\text {serv }}$ refers to the service bits and
TABLE I

SYSTEM PARAMETERS

\begin{tabular}{|c|c||c|c|}
\hline Parameter & Value & Parameter & Value \\
\hline$T_{\text {slot }}$ & $9 \mu s$ & $T_{h}$ & $10 \mathrm{~ms}$ \\
\hline$T_{S I F S}$ & $10 \mu s$ & $L_{\text {serv }}$ & $16 \mathrm{~b}$ \\
\hline$T_{D I F S}$ & $28 \mu s$ & $L_{\text {tail }}$ & $6 \mathrm{~b}$ \\
\hline$T_{E I F S}$ & $88 \mu s$ & $L_{R T S}$ & $20 \mathrm{~B}$ \\
\hline$C W_{\min }$ & 15 & $L_{C T S} L_{A C K}$ & $14 \mathrm{~B}$ \\
\hline$C W_{\max }$ & 1023 & $L_{M A C h d r}$ & $34 \mathrm{~B}$ \\
\hline$T_{B O}$ & $67.5 \mu s$ & $L_{X O R h d r}$ & $40 \mathrm{~B}$ \\
\hline$T_{\text {pre }}$ & $16 \mu s$ & $L_{F C S}$ & $4 \mathrm{~B}$ \\
\hline$T_{\text {sig }}$ & $4 \mu s$ & $P_{t}$ & $1.65 \mathrm{~W}$ \\
\hline$T_{\text {sym }}$ & $4 \mu s$ & $P_{r}$ & $1.4 \mathrm{~W}$ \\
\hline$T_{\text {sigEx }}$ & $6 \mu s$ & $P_{i}$ & $1.15 \mathrm{~W}$ \\
\hline
\end{tabular}

$L_{\text {tail }}$ is the sequence of tail bits. $L_{x}$ is the packet length, where $x$ may be the RTS, CTS, ACK, or MAC data packet or MAC Protocol Data Unit (MPDU). The MPDU includes a frame body or MAC Service Data Unit (MSDU) with a MAC header $\left(L_{M A C h d r}\right)$ and a Frame Check Sequence (FCS) $\left(L_{F C S}\right)$. Also, a XOR header $\left(L_{X O R h d r}\right)$ is added to coded data packets [2]. Note that control response packets such as CTS and ACK are transmitted using the basic rates 6, 12, and $24 \mathrm{Mbps}$, based on the rate selecion rules specified in [3]. All the above parameters and their values are provided in Table I.

\section{B. Energy Efficiency and Energy Consumption}

The energy efficiency of protocol $x\left(\eta_{x}\right)$ is defined as the amount of bits contained in a MSDU $\left(L_{M S D U}\right)$ divided by the energy consumed $\left(E_{x}\right)$ to transmit the data packet that includes the MSDU:

$$
\eta_{x}[\mathrm{Mb} / \mathrm{J}]=\frac{8 \cdot L_{M S D U}}{E_{x}}
$$

where $E_{x}$ is defined as the product of power consumed $(P)$ and time spent $(T)$ in transmission and is split into three energy components, namely, transmitting $\left(E_{t}\right)$, receiving and overhearing $\left(E_{r}\right)$, and idle $\left(E_{i}\right)$. Let $P_{t}, P_{r}$, and $P_{i}$ denote the power consumed in each of those operational states. $T_{D I F S}$ and $T_{S I F S}$ are the DIFS and SIFS intervals, respectively. Since we consider no collisions, the backoff period $\left(T_{B O}\right)$ is an average value obtained from the minimum $\mathrm{CW}$ size $\left(C W_{\text {min }}\right)$ and the slot time $\left(T_{\text {slot }}\right)$ as $T_{B O}=\left(\frac{C W_{\min }}{2}\right) T_{\text {slot }}$.

In each transmission cycle of DCF, the transmitter and the receiver, respectively, consume energy to transmit and receive the RTS packet and the data packet and to receive and transmit the CTS packet and the ACK packet. Meanwhile, the $N-1$ source nodes not involved in the transmission consume energy to overhear the exchange of packets except for one that can only overhear the packets sent by the relay node. The relay node and the $N$ source nodes also consume energy for listening to the wireless channel for DIFS, average backoff, and SIFS intervals. In addition, one source node is idle when one of the other source nodes is transmitting to the relay node.

The energy consumption of DCF $\left(E_{D C F}\right)$ shows a minimum value and a higher stable value under saturation due to the long-term fairness characteristic. To compute the minimum energy consumption of DCF $\left(E_{D C F}^{\min }\right)$, we analyze the case when the source nodes can transmit $N$ packets and the relay 
node can forward all of them to their respective destinations. In contrast, the saturation energy efficiency of DCF $\left(E_{D C F}^{s a t}\right)$ is obtained when the relay node can perform data transmission only once every $N$ transmissions from the source nodes. Therefore, $E_{D C F}$ is given by

$$
\begin{aligned}
E_{D C F} & =\alpha\left(E_{t}+E_{r}+E_{i}\right) \\
E_{t} & =\beta\left(T_{R T S}+T_{C T S}+T_{D A T A}+T_{A C K}\right) P_{t} \\
E_{r} & =\left(\gamma\left(T_{R T S}+T_{D A T A}\right)+\delta\left(T_{C T S}+T_{A C K}\right)\right) P_{r} \\
E_{i} & =\epsilon\left(T_{D I F S}+T_{B O}+3 T_{S I F S}\right) P_{i} \\
& +\left(\zeta\left(T_{R T S}+T_{D A T A}\right)+\kappa\left(T_{C T S}+T_{A C K}\right)\right) P_{i}
\end{aligned}
$$

where $\alpha=\frac{1}{N}, \beta=2 N, \gamma=\delta=(2 N-1) N^{2}, \epsilon=2 N(N+1)$, and $\zeta=\kappa=N^{2}$ for $E_{D C F}^{\min }$ and $\alpha=1, \beta=N+1, \gamma=N(N-1)+N$, $\delta=N^{2}+N-1, \epsilon=(N+1)^{2}, \zeta=N$, and $\kappa=1$ for $E_{D C F}^{s a t}$.

The energy consumption of COPE ( $\left.E_{C O P E}\right)$ can be derived in a similar way to that of DCF. The minimum energy consumption of COPE $\left(E_{C O P E}^{\min }\right)$ is calculated when the source nodes perform $N$ transmissions and the relay node forwards $N / 2$ coded packets. On the contrary, the saturation energy consumption $\left(E_{C O P E}^{s a t}\right)$ is computed considering that the relay node can only send a coded packet once every $N$ transmissions from the source nodes. Thus, $E_{C O P E}$ is expressed as

$$
\begin{aligned}
E_{C O P E} & =\alpha\left(E_{t}+E_{r}+E_{i}\right) \\
E_{t} & =\left(\beta\left(T_{R T S}+T_{C T S}+T_{A C K}\right)+N T_{D A T A}\right) P_{t} \\
& +\gamma T_{X O R D A T A} P_{t} \\
E_{r} & =\left(\delta T_{R T S}+N(N-1) T_{D A T A}+\epsilon\left(T_{C T S}+T_{A C K}\right)\right) P_{r} \\
& +\zeta T_{X O R D A T A} P_{r} \\
E_{i} & =\kappa\left(T_{D I F S}+T_{B O}+3 T_{S I F S}\right) P_{i} \\
& +\left(N\left(T_{R T S}+T_{D A T A}\right)+T_{C T S}+T_{A C K}\right) P_{i}
\end{aligned}
$$

where $\quad \alpha=\frac{1}{N}, \quad \beta=N+\frac{N}{2}, \quad \gamma=\frac{N}{2}, \quad \delta=N(N-1)+\frac{N^{2}}{2}$, $\epsilon=N^{2}+\frac{N}{2}(N-1), \quad \zeta=\frac{N^{2}}{2}, \quad$ and $\quad \kappa=(N+1)\left(N+\frac{N}{2}\right)$ for $E_{C O P E}^{\min }$ and $\alpha=\frac{1}{2}, \quad \beta=N+1, \quad \gamma=1, \quad \delta=N(N-1)+N$, $\epsilon=N^{2}+N-1, \zeta=N$, and $\kappa=(N+1)^{2}$ for $E_{C O P E}^{s a t}$.

The energy consumption of BidMAC $\left(E_{B i d M A C}\right)$ is based on that of DCF with the following differences. The receiver and the transmitter consume energy to transmit and receive a data packet (without an ACK packet) and to receive and transmit an ACK packet, respectively. In addition, the other $N-1$ source nodes consume energy to overhear the data packet from the receiver. The relay node and the $\mathrm{N}$ source nodes also consume energy for an additional SIFS interval. Note that in BidMAC the relay node is able to forward $N$ packets form end to end in $N$ transmission slots gained by the source nodes. As a result, $E_{B i d M A C}$ is written as

$$
\begin{aligned}
E_{\text {BidMAC }} & =\frac{1}{N}\left(E_{t}+E_{r}+E_{i}\right) \\
E_{t} & =N\left(T_{R T S}+T_{C T S}+T_{A C K}+2 T_{D A T A}\right) P_{t} \\
E_{r} & =N\left((N-1)\left(T_{R T S}+T_{A C K}\right)+N T_{C T S}\right) P_{r} \\
& +(N+N-1) N T_{D A T A} P_{r} \\
E_{i} & =(N+1) N\left(T_{D I F S}+T_{B O}+4 T_{S I F S}\right) P_{i} \\
& +N\left(T_{R T S}+T_{D A T A}+T_{A C K}\right) P_{i}
\end{aligned}
$$

Finally, the energy consumption of BidCode $\left(E_{\text {BidCode }}\right)$ consists of several parts of the energy components of the previously analyzed protocols. In BidCode, the source nodes perform $N$ data transmissions and the relay node only requires $\mathrm{N} / 2$ coded data transmissions using reverse direction communication to exchange $N$ packets from end to end. Hence, $E_{\text {BidCode }}$ is represented by

$$
\begin{aligned}
E_{\text {BidCode }} & =\frac{1}{N}\left(E_{t}+E_{r}+E_{i}\right) \\
E_{t} & =N\left(T_{R T S}+T_{C T S}+T_{A C K}+T_{D A T A}\right) P_{t} \\
& +(N / 2) T_{X O R D A T A} P_{t} \\
E_{r} & =N(N-1)\left(T_{R T S}+T_{D A T A}\right) P_{r} \\
& +\left(N T_{C T S}+(N / 2) T_{X O R D A T A}\right) N P_{r} \\
& +\left(\left(N^{2} / 2\right)+(N / 2)(N-1)\right) T_{A C K} P_{r} \\
E_{i} & =(N+1)\left(N\left(T_{D I F S}+T_{B O}\right)+7(N / 2) T_{S I F S}\right) P_{i} \\
& +\left(\left(T_{R T S}+N T_{D A T A}\right)+(N / 2) T_{A C K}\right) P_{i}
\end{aligned}
$$

\section{Evaluation Results}

The energy efficiency results of DCF, COPE, BidMAC, and BidCode are presented in this section by means of the analysis presented in the previous section and simulations based on a custom-made simulator in Python.

We simulate the Alice and Bob and cross scenarios shown in Fig. 1. All the nodes are static and within the transmission range of each other except one that is hidden from each source node. However, we assume that, although they cannot properly receive data, a pair of source nodes hidden from each other are at least able to perform carrier sensing when one of them is transmitting. The source nodes generate data packets of constant length through a Poisson arrival process. The relay node does not generate own traffic but only forwards the received packets to their respective destinations.

The system parameters and their values are provided in Table I. The Extended Inter Frame Space (EIFS) is the time that follows a collision of packets and is calculated as $T_{E I F S}=T_{D I F S}+T_{S I F S}+T_{A C K}(6 \mathrm{Mbps})$. The holding time $\left(T_{h}\right)$ specifies how much time a relay node can store a packet before forwarding it without coding in order to increase coding opportunities. All simulation runs were repeated 10 times for the duration of $20 \mathrm{~s}$ each and the simulation results in the plots are obtained with a $95 \%$ confidence interval lower than 0.05 .

Fig. 3 shows the network energy efficiency of the protocols considering different values for the traffic load, MSDU length, and PHY data rate in the two reference scenarios.

Figs. 3a and $3 \mathrm{~d}$ show that for high traffic loads COPE and BidCode outperform DCF and BidMAC in the Alice and Bob scenario whereas BidCode and BidMAC perform the best in the cross scenario. In the first case, the reason is that COPE and BidCode reduce the number of data transmissions and therefore nodes consume less energy for transmitting and receiving packets when compared to DCF and BidMAC. In the second case, the reason is that, unlike DCF and COPE, BidMAC and BidCode are not affected by the MAC fairness, hence providing a higher amount of channel access opportunities 


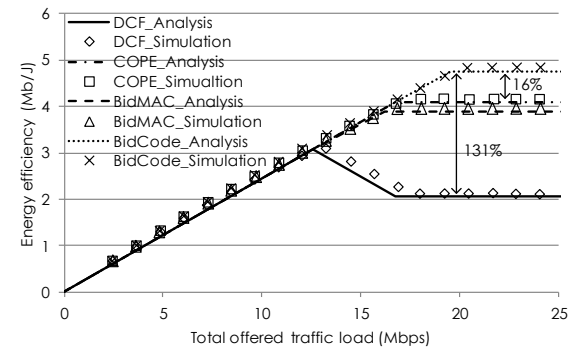

(a) A\&B: Energy efficiency vs. Load.

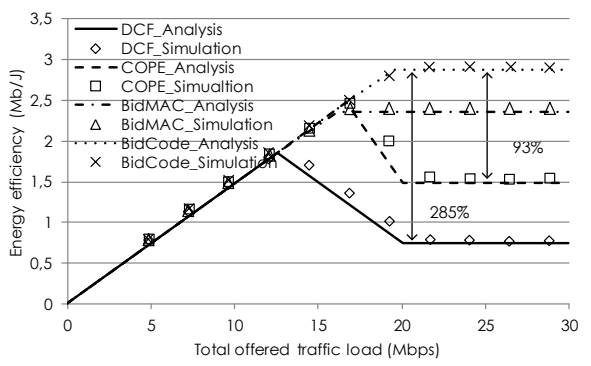

(d) Cross: Energy efficiency vs. Load.

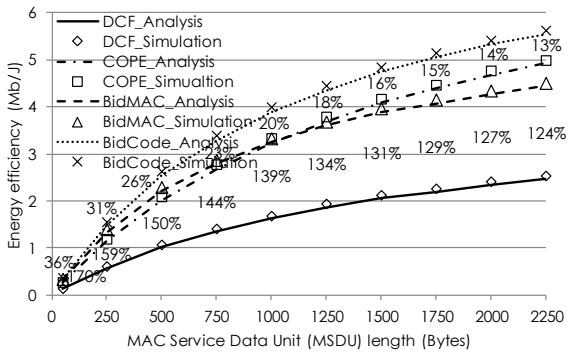

(b) A\&B: Energy efficiency vs. MSDU.

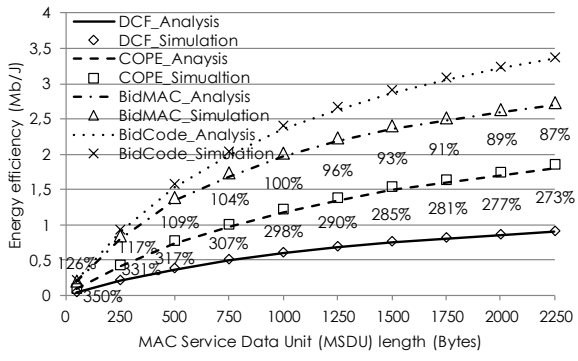

(e) Cross: Energy efficiency vs. MSDU.

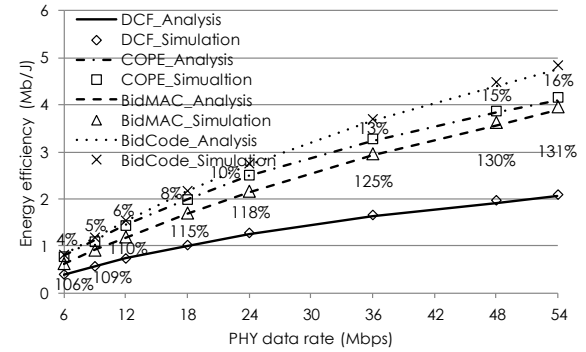

(c) A\&B: Energy efficiency vs. Rate.

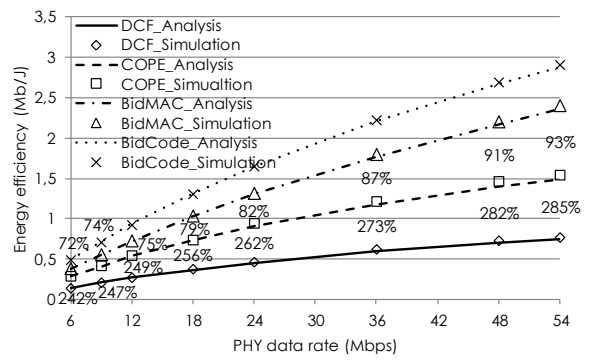

(f) Cross: Energy efficiency vs. Rate.

Fig. 3. Energy efficiency of DCF, COPE, BidMAC, and BidCode as a function of the traffic load, MSDU length (1500 bytes), and data rate (54 Mbps) in the Alice and Bob (A\&B) and cross topologies.

for the relay node. For this reason, the gains of BidCode versus DCF $(131 \%, 285 \%)$ and versus COPE $(16 \%, 93 \%)$ are significantly higher in the cross scenario.

The results of Figs. $3 \mathrm{~b}$ and $3 \mathrm{e}$ show that, while BidCode performs the best in both scenarios for all packet lengths, BidMAC does not outperform COPE in the Alice and Bob scenario for long packet lengths. The reason is that in BidMAC, and also in BidCode, the impact of the reduced overhead time of control packets and silent periods on the total transmission time is lower when the data transmission time is longer. For this reason, the gains of BidCode versus DCF (170-124\%, 350-273\%) and versus COPE (36-13\%, 126-87\%) decrease as the packet length increases in both scenarios. Similarly, the results in Figs. $3 \mathrm{c}$ and $3 \mathrm{f}$ show that BidCode achieves the highest energy efficiency for all data rates. Note that higher gains versus DCF $(106-131 \%, 242-285 \%)$ and versus COPE $(4-16 \%, 72-93 \%)$ are given for faster rates in both scenarios.

\section{CONCLUSiOnS}

This paper has comprehensively evaluated via analysis and simulation the energy efficiency of BidCode, a new NC-aware MAC protocol for wireless networks based on IEEE 802.11. Two well-known network topologies have been considered in the evaluation, namely, Alice and Bob, i.e., two source nodes and a relay node, and cross, i.e., four source nodes and a relay node. Also, important system parameters like the traffic load, packet length, and data rate, have been taken into account to compare BidCode with DCF and DCF+NC, i.e., COPE. The evaluation results have shown that BidCode is the most energyefficient solution especially in the cross scenario and for high traffic loads, short packet lengths, and fast data rates.

In order to validate the high performance of BidCode in a more realistic environment, ongoing work is aimed at implementing BidCode in several programmable wireless platforms and measuring the network throughput and energy efficiency.

\section{REFERENCES}

[1] R. Ahlswede, N. Cai, S.-Y. Li, and R. Yeung, "Network information flow," IEEE Transactions on Information Theory, vol. 46, no. 4, pp. 1204-1216, 2000.

[2] S. Katti, H. Rahul, W. Hu, D. Katabi, M. Médard, and J. Crowcroft, "XORs in the Air: Practical Wireless Network Coding," in ACM SIGCOMM, 2006, pp. 243-254.

[3] IEEE, Part 11: Wireless LAN Medium Access Control (MAC) and Physical Layer (PHY) Specifications, IEEE 802.11 Std., 2012.

[4] F. Xie, L. Du, Y. Bai, and L. Chen, "Popularity Aware Scheduling for Network Coding based Content Distribution in Ad Hoc Networks," in IEEE PIMRC, 2007, pp. 1-5.

[5] D. Umehara, C. Huang, S. Denno, M. Morikura, and T. Sugiyama, "Enhancement of IEEE 802.11 and Network Coding for Single-Relay Multi-User Wireless Networks," in IEEE ICSPCS, 2010, pp. 1-9.

[6] N. De Coppi, J. Ning, G. Papageorgiou, M. Zorzi, S. Krishnamurthy, and T. La Porta, "Network Coding Aware Queue Management in Multi-Rate Wireless Networks," in IEEE ICCCN, 2012, pp. 1-7.

[7] A. Paramanathan, P. Pahlevani, D. E. Lucani, and F. H. P. Fitzek, "On the Need of Novel Medium Access Control Schemes for Network Coding enabled Wireless Mesh Networks," in IEEE ICC, 2013, pp. 1-6.

[8] R. Palacios, F. Granelli, A. Paramanathan, J. Heide, and F. H. P. Fitzek, "Coding-aware MAC: Providing Channel Access Priority for Network Coding with Reverse Direction DCF for IEEE 802.11-based Wireless Networks," in IEEE ICC, 2014, pp. 1278-1283.

[9] R. Palacios, F. Granelli, D. Gajic, and A. Foglar, "An Energy-Efficient MAC Protocol for Infrastructure WLAN Based on Modified PCF/DCF Access Schemes Using a Bidirectional Data Packet Exchange," in IEEE CAMAD, 2012, pp. 216-220.

[10] R. Palacios, F. Granelli, D. Kliazovich, L. Alonso, and J. AlonsoZarate, "Energy Efficiency of an Enhanced DCF Access Method Using Bidirectional Communications for Infrastructure-based IEEE 802.11 WLANs," in IEEE CAMAD, 2013, pp. 38-42.

[11] R. Palacios, H. Haile, J. Alonso-Zarate, and F. Granelli, "Analysis of a Network Coding-Aware MAC Protocol for IEEE 802.11 Wireless Networks with Reverse Direction Transmissions," in IEEE GLOBECOM, 2014, accepted for publication. 